\title{
Visit-to-Visit Blood Pressure
}

\section{Variability Are Associated with}

\section{an Imbalance between Sympathetic and Parasympathetic Tone in Hypertensive Patients}

\author{
Takuzo Hano1*, Yumi Koike ${ }^{2 \#}$ \\ ${ }^{1}$ Satellite Clinic for Integrative and Anti-Aging Medicine, Wakayama Medical University, Wakayama, Japan \\ ${ }^{2}$ Department of Rehabilitation Medicine, School of Medicine, Wakayama Medical University, Wakayama, Japan \\ Email: hanotaku@wakayama-med.ac.jp, koikoi@wakayama-med.ac.jp
}

How to cite this paper: Hano, T. and Koike, Y. (2022) Visit-to-Visit Blood Pressure Variability Are Associated with an Imbalance between Sympathetic and Parasympathetic Tone in Hypertensive Patients. Health, 14, 246-253.

https://doi.org/10.4236/health.2022.142019

Received: January 26, 2022

Accepted: February 22, 2022

Published: February 25, 2022

Copyright $\odot 2022$ by author(s) and Scientific Research Publishing Inc. This work is licensed under the Creative Commons Attribution International License (CC BY 4.0).

http://creativecommons.org/licenses/by/4.0/

\begin{abstract}
Greater fluctuations in office blood pressure increase the risk of stroke and blood pressure volatility is an important risk factor for cardiovascular events. Effects of sympathetic and parasympathetic nerve on blood pressure regulation are well known, however, those on visit-to visit variation remains unclear. Aim of this study is to clarify the contribution of sympathetic and parasympathetic balance on blood pressure fluctuation. Methods: We enrolled 23 outpatients with essential hypertension. We measured blood pressure, and pulse rate at clinic 14 times in a row and calculated coefficient of variance (CV) as visit-to-visit variability. The velocity of pupil contraction, miosis (VC) and dilation, mydriasis (VD) was determined from pupillary function test. Results: Systolic blood pressure and diastolic blood pressure were not significantly correlated with VC, VD and VD/VC. Heart rate was not correlated with VC and VD, but significantly correlated with VD/VC. CV of systolic blood pressure, diastolic blood pressure and heart rate were not significantly correlated with VC or VD. Ratio of VD to VC significantly negative correlated with $\mathrm{CV}$ of blood pressure, diastolic blood pressure, and heart. Value of VD was significantly correlated with value of VC. Conclusion: Visit-to-visit variability of blood pressure and heart rate is not related with sympathetic or parasympathetic tone independently but augmented by the impaired sympathetic and parasympathetic balance.
\end{abstract}




\section{Keywords}

Visit-to-Visit Variability, Blood Pressure, Sympathetic Activity, Parasympathetic Activity

\section{Introduction}

Visit-to-visit variability of systolic blood pressure and maximal systolic blood pressure in patients with transient ischemic attack are risk factors for stroke, independent of mean blood pressure, and its importance has recently been reported [1] [2]. It has also been reported that greater fluctuations in-office blood pressure increase the risk of stroke after adjusting blood pressure levels and other recreational factors, and blood pressure volatility is an important risk factor for cardiovascular events [2].

It is well known that sympathetic and parasympathetic nerve affects blood pressure regulation. Sympathetic nerves are an important factor in short-term blood pressure regulation and resting sympathetic nerve activity is associated with daytime home and office blood pressure fluctuations and nocturnal blood pressure reduction [3]. Beta blockers, which are sympathomimetics, are known to suppress short-term fluctuations in blood pressure and heart rate [4]. On the other hand, it has been reported that the difference between the basal blood pressure measurement value and the maximum blood pressure measurement value decreases in patients taking $\beta$-blockers, but there is an inverse correlation between changes in blood pressure fluctuations and changes in blood noradrenaline [5]. These results show that sympathetic nerves are associated with shortterm blood pressure fluctuations, but there are many unclear points regarding the relationship between blood pressure visit-to-visit variability. In addition, we have conducted research to evaluate autonomic nerve function by introducing a method for non-invasively measuring sympathetic nerve function and parasympathetic nerve function using a pupil function test [6] [7]. In this study, we analyzed the relationship between hypertension visit-to-visit variability and sympathetic and parasympathetic nerve activity obtained from pupil function tests and clarify the involvement of autonomic nerves visit-to-visit variability in hypertension.

\section{Subjects and Methods}

\subsection{Subjects}

We enrolled 23 outpatients ( 12 males, 11 females) with mild essential hypertension who had been administered antihypertensive drugs for at least six months at Wakayama Medical University Satellite Clinic. Average age was $65.9 \pm 11.7$, mean \pm SD. Single therapy of calcium antagonist was performed for seven patients, combination of calcium antagonist and angiotensin receptor blockade for 6patients, combination of calcium antagonist, angiotensin receptor blockade and 
diuretics for 5 patients, and combination of calcium antagonist and diuretics for one patient. Patients with secondary hypertension, diabetes, cataract, or other medical problems requiring specific treatments were excluded. All 23 patients provided written, informed consent to participate in this study. The Ethics Committee at Wakayama Medical University approved this study (\#878), which conformed to the principles outlined in the Declaration of Helsinki.

\subsection{Experimental Protocol}

Pupillary function tests proceeded under similar environmental conditions between 09:00 and 12:00. After resting for 15 minutes, the patients underwent pupil function tests. We measured systolic and diastolic blood pressure, and pulse rate at clinic 14 times in a row and calculated coefficient of variance (CV) as visit-to-visit variability.

\subsection{Pupillary Function Test}

Parameters of the pupillary light reflex were examined using pupillometry in low light conditions at rest. The patients rested for $15 \mathrm{~min}$ to acclimate their eyes to low light levels. The experimental conditions were explained to each patient in detail and then pupillometry of both eyes proceeded. The detailed method was described in the previous report [6] [7]. In brief, the diameter of the pupil was measured using an Iriscorder Dual C10641 pupillometer (Hamamatsu Photonics, Hamamatsu, Japan). The velocity of pupil contraction, miosis (VC) and dilation, mydriasis (VD) was determined from the total of pupillary function. We used $\mathrm{VD}$ as marker of sympathetic activity and VC as marker of parasympathetic activity. And calculated VD/VC ratio as balance of sympathetic and parasympathetic activity.

\subsection{Statistical Analysis}

A single correlation coefficient between coefficient of variation of blood pressure or heart rate and VD, VC, and VD/VC ratio was calculated using IBM SPSS 26.

Data of each parameter were expressed as means \pm SD. $\mathrm{P}<0.05$ was taken to indicate a significant difference.

\section{Results}

The average age is $66 \pm 6$ years old. Average of systolic blood pressure, diastolic blood pressure , and heart rate were $122.4 \pm 32.7 \mathrm{mmHg}, 72.7 \pm 20.0 \mathrm{mmHg}$, and $60.7 \pm 18.0$ /minutes, respectively. The coefficient of variation for systolic blood pressure, diastolic blood pressure, and heart rate was $5.57 \pm 2.13,6.78 \pm 2.96$ and $6.73 \pm 2.79$, respectively. VC, $\mathrm{VD}$, and $\mathrm{VD} / \mathrm{VC}$ were $3.29 \pm 0.79 \mathrm{~ms}, 1.69 \pm 0.58$ ms., and $0.52 \pm 0.14$. There was no difference between female and male values.

Systolic blood pressure and diastolic blood pressure were not significantly correlated with VC, VD and VD/VC. Heart rate was not correlated with VC and $\mathrm{VD}$, but significantly correlated with VD/VC $(\mathrm{p}=0.032)$. 
Correlation coefficient between coefficient of variation of systolic blood pressure, diastolic blood pressure and heart rate were not significantly correlated with VC and VD. On the other hand, there was a significant negative correlation between coefficient of variation of systolic blood pressure $(p=0.018)$, diastolic blood pressure $(P<0.001)$, and heart rate $(\mathrm{p}=0.007)$ and ratio of VD/VC (Table 1, Figures 1-3). Value of VD was significantly correlated with value of VC $(\mathrm{p}=0.008$, Figure 4$)$.

Table 1. Correlationship between coefficient of variation of blood pressure and parameters of pupillary function test.

\begin{tabular}{|c|c|c|c|c|c|c|c|c|c|c|c|c|}
\hline \multirow[b]{2}{*}{ Correlation coefficient } & \multicolumn{2}{|c|}{ Systolic BP } & \multicolumn{2}{|c|}{$\begin{array}{c}\text { CV of } \\
\text { systolic BP }\end{array}$} & \multicolumn{2}{|c|}{ Diastolic BP } & \multicolumn{2}{|c|}{$\begin{array}{c}\mathrm{CV} \text { of } \\
\text { diastolic BP }\end{array}$} & \multicolumn{2}{|c|}{ Heart rate } & \multicolumn{2}{|c|}{$\begin{array}{c}\mathrm{CV} \text { of } \\
\text { heart rate }\end{array}$} \\
\hline & $\mathrm{R}^{2}$ & p-value & $\mathrm{R}^{2}$ & p-value & $\mathrm{R}^{2}$ & $\mathrm{p}$-value & $\mathrm{R}^{2}$ & p-value & $\mathrm{R}^{2}$ & p-value & $\mathrm{R}^{2}$ & $\mathrm{p}$-value \\
\hline Velocity of miosis (VC) & 0.090 & 0.170 & 0.001 & 0.865 & 0.095 & 0.153 & 0.105 & 0.141 & 0.021 & 0.530 & 0.062 & 0.250 \\
\hline Velocity of midriasis (VD) & 0.001 & 0.893 & 0.165 & 0.054 & 0.013 & 0.609 & 0.161 & 0.065 & 0.146 & 0.087 & 0.073 & 0.198 \\
\hline $\mathrm{VD} / \mathrm{VC}$ & 0.087 & 0.173 & 0.238 & $0.018^{*}$ & 0.019 & 0.527 & 0.428 & $0.001^{*}$ & 0.219 & $0.032^{*}$ & 0.300 & $0.007^{\star}$ \\
\hline
\end{tabular}

VC: velocity of pupil contraction (miosis), VD: velocity of pupil dilation, mydriasis (VD), CV: coefficient of variance. Systolic BP: average of systolic blood pressure, Dyastolic BP: average of systolic blood presuure, Heart rate: average of heart rate. ${ }^{\star}$ means as significant value of $\mathrm{p}<0.05$.

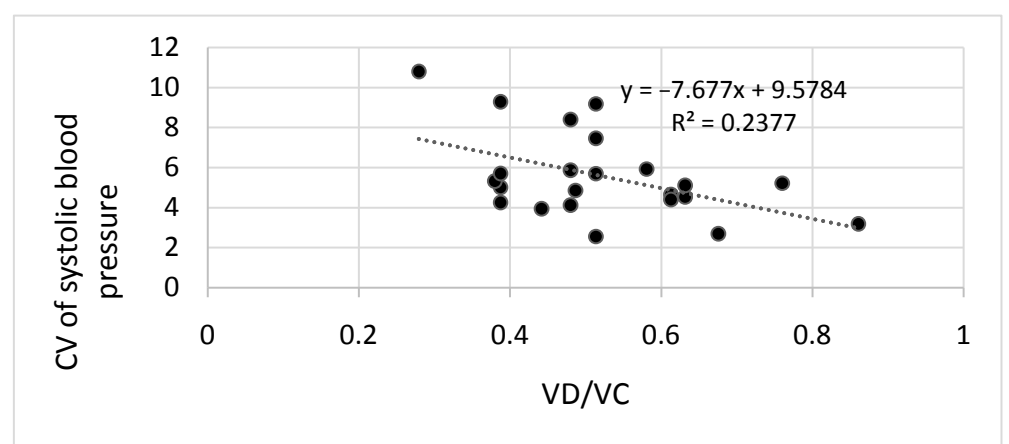

Figure 1. Relationship between VD/VC ratio and CV of systolic blood pressure. VC: velocity of pupil contraction (miosis); VD: velocity of pupil dilation, mydriasis (VD); CV: coefficient of variance.

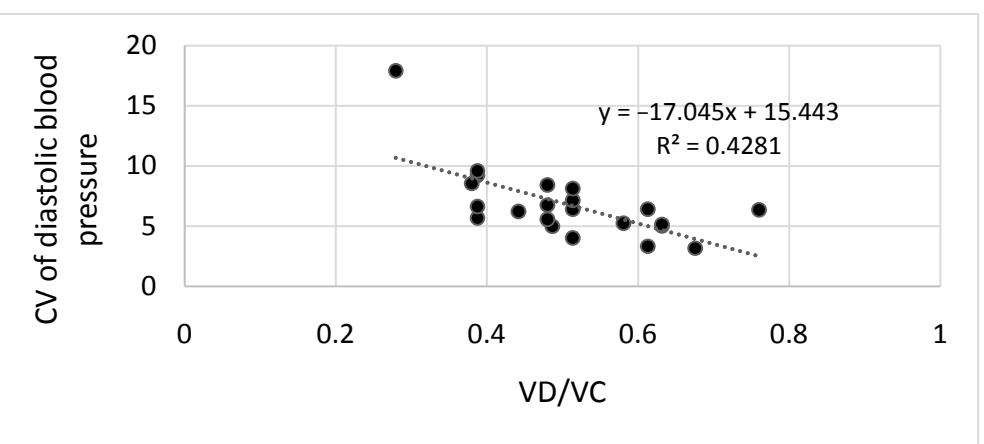

Figure 2. Relationship between VD/VC ratio and CV of diastolic blood pressure. VC: velocity of pupil contraction (miosis); VD: velocity of pupil dilation, mydriasis (VD); CV: coefficient of variance. 


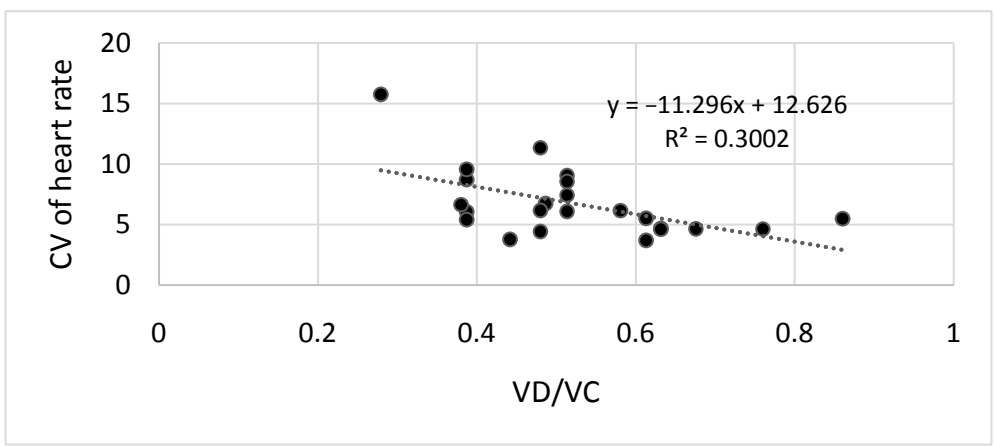

Figure 3. Relationship between VD/VC ratio and CV of heart rate. VC: velocity of pupil contraction (miosis); VD: velocity of pupil dilation, mydriasis (VD); CV: coefficient of variance.

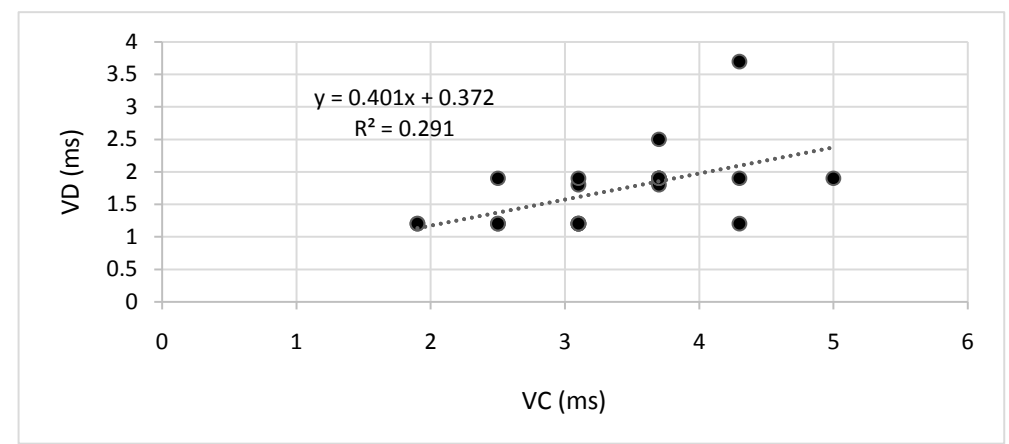

Figure 4. Relationship between VC and VD. VC: velocity of pupil contraction (miosis), VD: velocity of pupil dilation, mydriasis (VD).

\section{Discussion}

Several reports have shown that the visit-to-visit variability of blood pressure is an independent risk factor for cardiovascular events.

Rothwell PM showed that visit-to-visit systolic blood pressure variability and maximal SBP were strong predictors of stroke, independent of mean SBP, and increased residual variability of SBP in treated hypertensive patients was a risk of cardio-vascular events [1]. We found that blood pressure fluctuations from visit to visit helped prevent cardiovascular events and predict blood pressure fluctuations, as they are associated with high blood pressure. Hata $\mathrm{Y}$ also reported that fluctuations in clinic blood pressure were risk factors for stroke [2].

The sympathetic nervous system is one of the useful factors that cause blood pressure fluctuations and may be involved in the visit-to-visit variability of blood pressure.

Narkiewicz K showed that higher resting measurements of sympathetic traffic are associated with greater daytime blood pressure variability and a more marked nocturnal decline in blood pressure in normal subjects [3]. These findings suggest that sympathetic neural mechanisms may contribute importantly to the regulation of blood pressure over the 24-hour period. Sundlö G reported that the sympathetic outflow to skeletal muscles was important for buffering acute blood pressure changes but has little influence on the long-term blood pressure 
level [8].

Thus, it has been shown that sympathetic nerve hyperactivity in the acute phase affects short-term blood pressure fluctuations, but it is unclear whether it affects blood pressure visit-to-visit variability.

Olga V showed no correlation was found between blood pressure response to cold pressure and hand-grip test and ambulatory blood pressure, daily blood pressure variability [9]. In addition, Floras JS showed that ambulatory blood pressure was lower during beta-blockade treatment, whereas pulse interval, its variability, and baroreceptor reflex sensitivity were higher [10]. Thus, conflict observations have been reported regarding the relationship between blood pressure visit-to-visit variability and sympathetic nerve activity.

In clinical studies, plasma catecholamines, sympathetic skin response, and heart rate variability have generally been used for the assessment of sympathetic nerve activity. However, blood sampling for measurement of plasma catecholamines can change the levels of catecholamines, and a time lag exists between the physical loading and elevation of plasma catecholamines. It is also difficult to measure sympathetic and parasympathetic function non-invasively at the same time. For this reason, it was difficult to evaluate the relationship between the visit-to-visit variability of blood pressure and autonomic nerve function, but it is possible to evaluate sympathetic nerve and parasympathetic nerve function by pupil dilation and miosis speed by pupil function test. Pupillometry using light stimuli is a reproducible and non-invasive method for the assessment of autonomic function [11] [12] [13]. Moreover, pupillometry can evaluate sympathetic and parasympathetic function separately in a short time [11] [12]. Consequently, we used pupillometry to evaluate autonomic activity in this study as reported previous reports. Several reports indicated that pupillary autonomic function assessed by dynamic pupillometry correlated with cardiac autonomic functions [14]. Autonomic impairment was detected in diabetes and neurological diseases [13] [15] [16] [17] [18].

Our previous study [6] [7] evaluated elevated blood pressure and sympathetic and parasympathetic function by handgrip testing using pupillometry. The handgrip loading test showed an increase of VD associated with an increase of VC. It was considered that the short-term increase in sympathetic nerve function was compensatory suppressed by the increase in parasympathetic nerve activity. In the present study, value of VD was significantly correlated with value of VC. It was shown that resting sympathetic tone was generally synchronized with parasympathetic tone.

The present study showed that systolic blood pressure, diastolic blood pressure and pulse did not correlate with VD and VC. Systolic and diastolic blood pressure were not related with $\mathrm{VD} / \mathrm{VC}$ ratio, but heart rate was negatively correlated with VD/VC.

These data showed clinic blood pressure did not associate with neither sympathetic nor parasympathetic tone. On the other hand, heart rate was related 
with relatively weak sympathetic tone compared with parasympathetic tone.

In addition, the coefficient of variation of systolic, diastolic, and pulse did not correlate with either VD or VC alone. These data indicate that sympathetic and parasympathetic activity alone does not affect blood pressure fluctuations. On the other hand, coefficient of variation of systolic blood pressure, diastolic blood pressure and pulse rate showed a significant inverse correlation with the VD/VC ratio.

Recent data showed that sympathetic/parasympathetic coactivation is causally linked to changes in heart rate (HR) dynamics [19].

In conclusion, visit-to-visit variability of blood pressure and heart rate is augmented by the impaired sympathetic and parasympathetic coactivation. Furthermore, VD/VC ratio can be a useful tool to detect blood pressure and heart rate variability.

\section{Acknowledgements}

Grant-in-Aid for Scientific Research (C) (\#26460915) from the Ministry of Education, Culture, Sports, Science, and Technology (MEXT), Japan.

\section{Conflicts of Interest}

The authors declare no conflicts of interest regarding the publication of this paper.

\section{References}

[1] Rothwell, P.M., Howard, S.C., Dolan, E., O’Brien, E., Dobson, J.E., Dahlöf, B., Sever, P.S. and Poulter, N.R. (2010) Prognostic Significance of Visit-to-Visit Variability, Maximum Systolic Blood Pressure, and Episodic Hypertension. The Lancet, 375, 895905. https://doi.org/10.1016/S0140-6736(10)60308-X

[2] Hata, Y., Kimura, Y., Muratani, H., Fukiyama, K., Kawano, Y., Ashida, T., Yokouchi, M., Imai, Y., Ozawa, T., Fujii, J. and Omae, T. (2000) Office Blood Pressure Variability as a Predictor of Brain Infarction in Elderly Hypertensive Patients. Hypertension Research, 23, 553-560. https://doi.org/10.1291/hypres.23.553

[3] Narkiewicz, K., Winnicki, M., Schroeder, K., Phillips, B.G., Kato, M., Cwalina, E. and Somers, V.K. (2002) Relationship between Muscle Sympathetic Nerve Activity and Diurnal Blood Pressure Profile. Hypertension, 39, 168-172. https://doi.org/10.1161/hy1201.097302

[4] Girard, A., Meilhac, B., Mounier-Vehier, C. and Elghozi, J.L. (1995) Effects of BetaAdrenergic Blockade on Short-Term Variability of Blood Pressure and Heart Rate in Essential Hypertension. Clinical and Experimental Hypertension, 17, 15-27. https://doi.org/10.3109/10641969509087051

[5] de Leeuw, P.W., Falke, H.E., Kho, T.L., Vandongen, R., Wester, A. and Birkenhäger, W.H. (1977) Effects of Beta-Adrenergic Blockade on Diurnal Variability of Blood Pressure and Plasma Noradrenaline Levels. Acta Medica Scandinavica, 202, 389-392. https://doi.org/10.1111/j.0954-6820.1977.tb16849.x

[6] Koike, Y., Kawabe, T., Nishihara, K., Iwane, N. and Hano, T. (2015) Cilnidipine But Not Amlodipine Suppresses Sympathetic Activation Elicited by Isometric Exercise in Hypertensive Patients. Clinical and Experimental Hypertension, 37, 531-535. https://doi.org/10.3109/10641963.2015.1025959 
[7] Koike, Y., Kawabe, T., Nishihara, K. and Iwane, N. (2016) Hano Effects of Azelnidipine and Amlodipine on Exercise-Induced Sympathoexcitation Assessed by Pupillometry in Hypertensive Patients. Hypertension Research, 39, 863-867. https://doi.org/10.1038/hr.2016.94

[8] Sundlöf, G. and Wallin, B.G. (1978) Human Muscle Nerve Sympathetic Activity at Rest. Relationship to Blood Pressure and Age. The Journal of Physiology, 274, 621637. https://doi.org/10.1113/jphysiol.1978.sp012170

[9] Olga, V., Lucio, M., Giuseppe, G., Stefano, M. and Paolo, P. (1995) Blood Pressure Response to Stress Tests Does Not Reflect Blood Pressure Variability and Degree of Cardiovascular Involvement in Young Hypertensives. International Journal of Cardiology, 48, 303-310. https://doi.org/10.1016/0167-5273(94)02237-D

[10] Floras, J.S., Hassan, M.O., Jones, J.V., Osikowska, B.A., Sever, P.S. and Sleight, P. (1988) Factors Influencing Blood Pressure and Heart Rate Variability in Hypertensive Humans. Hypertension, 11, 273-281. https://doi.org/10.1161/01.HYP.11.3.273

[11] Fotiou, F., Fountoulakins, K.N., Goulas, A., Alexopoulos, L. and Palikaras, A. (2000) Automated Standardized Pupillometry with Optical Method for Purposes of Clinical Practice and Research. Clinical Physiology, 20, 336-347.

https://doi.org/10.1046/j.1365-2281.2000.00259.x

[12] Fotiou, F., Fountoulakins, K.N., Tsolaki, M., Goulas, A. and Palikaras, A. (2000) Changes in Pupil Reaction to Light in Alzheimer's Disease Patients: A Preliminary Report. International Journal of Psychophysiology, 37, 111-120. https://doi.org/10.1016/S0167-8760(00)00099-4

[13] Zafar, S.F. and Suarez, J.I. (2014) Automated Pupillometer for Monitoring the Critically Ill Patient: A Critical Appraisal. Journal of Critical Care, 29, 599-603. https://doi.org/10.1016/j.jcrc.2014.01.012

[14] Okutucu, S., Civelekler, M., Sabanoglu, C., Aparci, M., Dikmetas, O., Uzun, S., Aksoy, H., Sahin, O.F., Yetis Sayin, B. and Oto, A. (2016) Assessment of the Relationship between Dynamic Pupillometry and Exercise Heart Rate Recovery among Healthy Subjects. European Review for Medical and Pharmacological Sciences, 20, 1344-1349.

[15] Lowenstein, O. and Loewenfeld, I.E. (1958) Electronic Pupillography, a New Instrument and Some Clinical Applications. AMA Archives of Ophthalmology, 59, 352-363. https://doi.org/10.1001/archopht.1958.00940040058007

[16] Pina, S.J. and Halonen, J.P. (1994) Infrared Pupillometry in the Assessment of Autonomic Function. Diabetes Research and Clinical Practice, 26, 61-66. https://doi.org/10.1016/0168-8227(94)90140-6

[17] Keivanidou, A., Fotiou, D., Arnaoutoglou, C., Arnaoutoglou, M., Fotiou, F. and Karlovasitou, A. (2010) Evaluation of Autonomic Imbalance in Patients with Heart Failure: A Preliminary Study of Pupillomotor Function. Cardiology Journal, 17, 65-72.

[18] Jain, S., Siegle, G.J., Gu, C., Moore, C.G., Ivanco, L.S., Jennings, J.R., Steinhauer, S.R., Studenski, S. and Greenamyre, J.T. (2011) Autonomic Insufficiency in Pupillary and Cardiovascular Systems in Parkinson's Disease. Parkinsonism \& Related Disorders, 17, 119-122. https://doi.org/10.1016/j.parkreldis.2010.11.005

[19] Eickholt, C., Jungen, C., Drexel, T., Alken, F., Kuklik, P., Muehlsteff, J., Makimoto, H., Hoffmann, B., Kelm, M., Ziegler, D., Kloecker, N., Willems, S. and Meyer, C. (2018) Sympathetic and Parasympathetic Coactivation Induces Perturbed Heart Rate Dynamics in Patients with Paroxysmal Atrial Fibrillation. Medical Science Monitor, 24, 2164-2172. https://doi.org/10.12659/MSM.905209 